\title{
A case of wet purpura due to etoricoxib induced thrombocytopenia
}

\author{
Robin George Manappallil', Vinod Krishnan² \\ ${ }^{1}$ Consultant - Physician, Department of Medicine, National Hospital, ${ }^{2}$ Professor and Head, Department of Medicine, \\ K.M.C.T. Medical College, Calicut, Kerala, India
}

Etoricoxib is a selective cyclo-oxygenase-2 inhibitor, commonly used in treatment of rheumatoid arthritis, gout and back pain. Pretibial edema and erythema, gastric irritation and risks of cardiovascular and cerebrovascular thrombotic events are some of the reported adverse effects due to etoricoxib. This case report is regarding a patient who presented with wet purpura following etoricoxib intake. Drug induced thrombocytopenia is an unreported side effect of etoricoxib.

\section{Access this article online}

Website:

http://nepjol.info/index.php/AJMS

DOI: 10.3126/ajms.v7i5.14674

E-ISSN: 2091-0576

P-ISSN: 2467-9100

Key words: Etoricoxib, Drug induced thrombocytopenia, Wet purpura

\section{INTRODUCTION}

Etoricoxib is a cyclo-oxygenase-2 (COX-2) inhibitor, which works to relieve pain and inflammation. It is used to treat painful conditions such as arthritis, back pain and gout. The risk of stroke and myocardial infarction have been reported with COX-2 inhibitor usage. ${ }^{1,2}$ Other adverse events include gastric irritation and pretibial oedema. ${ }^{3,4}$ However, a drug induced thrombocytopenia by etoricoxib has rarely been reported.

\section{CASE REPORT}

The patient being reported is a 51 year old male, shopkeeper, who presented to the Medicine OPD with one episode of bleeding from the mouth. While he was watering his garden in the morning, he blew through the pipe hose to remove a block and recognised a bloody taste in his mouth. On looking in the mirror, he noticed bleeding from his tongue. He did not give any history of fever or any other bleeding manifestations. He was taking tablet etoricoxib $90 \mathrm{mg}$ twice daily for the past 7 days, as over the counter medication, for his lower back pain. He had been prescribed this drug for 3 days by an orthopaedic surgeon, about 1 year ago, for back pain. He is not a diabetic or hypertensive, and is not on any regular medications.
On examination, he was moderately built and nourished; conscious, oriented and afebrile. His vitals were stable, with heart rate of 70 beats/minute, blood pressure of $110 / 70 \mathrm{mmHg}$ with no postural drop and respiratory rate of 20 breaths/minute. His tongue had a blackish discoloration, suggestive of wet purpura (Figure 1). There were petechial rashes and ecchymosis over upper and lower limbs (Figure 2). His systemic examinations were normal.

His complete blood count showed platelets of $8000 / \mu \mathrm{L}$ (150,000-450,000) and $\mathrm{Hb} 11 \mathrm{~g} \%$ (12-15), but no leucopenia. His electrolytes, renal and liver functions were normal. Prothrombin time and activated partial thromboplastin time were normal. Dengue serology, malarial smear and blood cultures were negative. Peripheral smear showed severe thrombocytopenia with normal platelet and red blood cell morphology, with no evidence of haemoparasites and abnormal cells. Viral markers for hepatitis B, hepatitis C and HIV were negative. Serology for Helicobacter pylori was negative. Antinuclear antibody and anti dsDNA, Coombs' test (direct and indirect) and serum protein electrophoresis were also negative. His $\operatorname{IgA}$ levels were normal. His chest Xray, ECG and ultrasound abdomen were normal. Urine microscopy did not showed any evidence of hematuria.

Based on the history of development of symptoms following 7 days of etoricoxib intake and history of its usage in the past, 


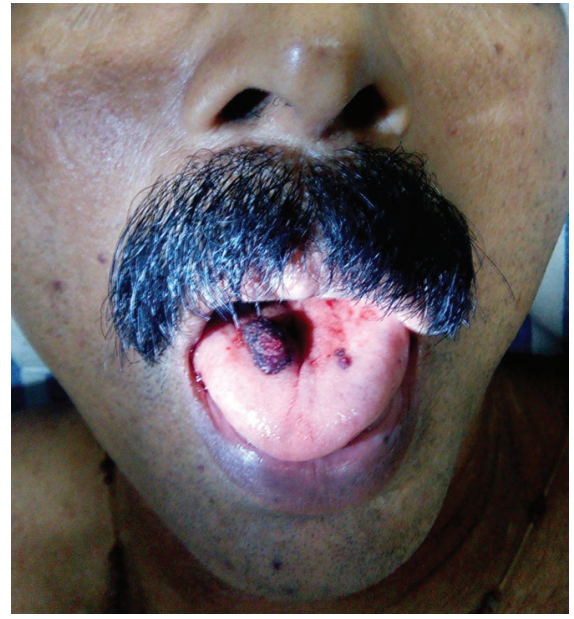

Figure 1: Wet purpura over the tongue

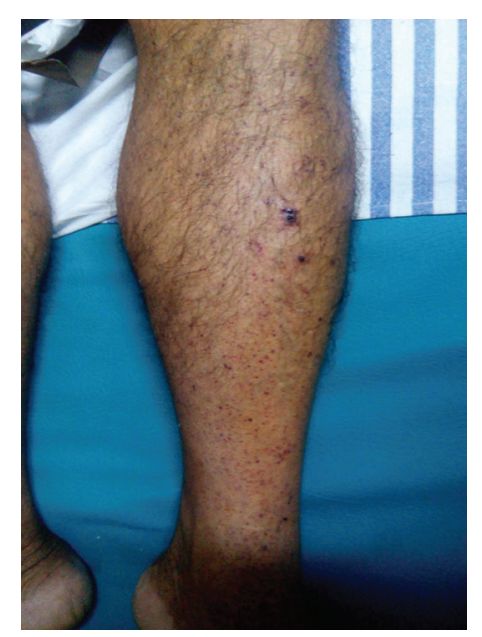

Figure 2: Petechiae and ecchymosis over lower limb

the diagnosis of drug induced immune thrombocytopenia was considered. In view of severe thrombocytopenia, he was given 4 units of platelet transfusion. He was also put on twice daily dose of injection pantoprazole. On day 3 , in spite of the transfusions and withholding of etoricoxib, his platelet increased only to $9000 / \mu \mathrm{L}$. He was given another 4 units of platelets the next day, but his platelet count continued to be almost the same. He did not have any new bleeding manifestations following admission. He was given intravenous methylprednisolone for 2 days. On day 5, his platelet counts increased to $13,000 / \mu \mathrm{L}$. He was started on tablet prednisolone $1 \mathrm{mg} / \mathrm{kg}$ once daily. Over the next 5 days, his platelet levels normalized without any need for further transfusions. His wet purpura disappeared and petechial rash and ecchymosis also started subsiding. He was discharged on tapering doses of prednisolone and reviewed after 1 week and 3 weeks of stopping prednisolone. His complete blood count was perfectly normal on both occasions. Since the patient had only thrombocytopenia, which had normalized, in response to steroids, bone marrow examination was avoided.

\section{DISCUSSION}

Platelets play a key role in maintaining hemostasis. The average life span of platelets is 7-10 days. ${ }^{5}$ Thrombocytopenia is defined as a platelet count less than $150,000 / \mu \mathrm{L} .{ }^{6}$ It is caused either due to decreased bone marrow production or sequestration or increased platelet destruction. A platelet count of 5000 to 10,000 is required to maintain the vascular integrity of microcirculation. Petechiae are the first to appear when there is a drastic fall in platelet count. The common sites are ankles and feet. Wet purpura and blood blisters in oral mucosa are indicators of risk of life threatening haemorrhage. ${ }^{5}$

Immune or idiopathic thrombocytopenic purpura (ITP) is an acquired disorder characterized by thrombocytopenia. It may be primary or secondary. Primary ITP is seen in both adults and children. Secondary ITP occurs in lymphoproliferative disorders, systemic lupus erythematosis and antiphospholipid antibody syndrome. It is also seen in association with infections like hepatitis B and C, HIV, and Helicobacter pylori. The administration of certain drugs can also cause thrombocytopenia. ${ }^{6}$

Drug induced thrombocytopenia (DITP) includes thrombocytopenia induced by drugs, beverages, foods and herbal products. There are several mechanisms leading to DITP. Heparin induced thrombocytopenia is one of the commonest forms, where there is thrombosis rather than bleeding. ${ }^{7}$ Drugs like quinine cause immune thrombocytopenia, where drug dependent antibodies are formed that react directly with the sensitizing drug to form an immune complex; which in turn reacts with the platelets causing its destruction. ${ }^{8}$ Certain drugs like penicillin trigger a humoral immune response only when they are linked to a macromolecule such as a protein, which act as a hapten. ' Tirofiban and eptifibatide are GPIIb/IIIa inhibitors that can cause thrombocytopenia by formation of antibodies that recognize GPIIb/IIIa in a complex with the particular ligand-mimetic. ${ }^{10}$ Medications like L-dopa, penicillamine and sulfamethoxazole trigger the production of platelet-specific autoantibodies, leading to a clinical picture similar to autoimmune thrombocytopenia. ${ }^{11}$ Chemotherapy agents cause a decrease in platelet production by generalized myelosuppression, ${ }^{12}$ while drugs like bleomycin can cause non-immune platelet destruction. ${ }^{13}$ Thiazide diuretics can cause thrombocytopenia either by selective suppression of megakaryocyte production or by an immune mediated mechanism. ${ }^{14}$

DITP should be suspected in cases of unexpected severe thrombocytopenia as well as recurrent thrombocytopenia with prompt recovery. Several drugs causing thrombocytopenia have been listed and reviewed systematically, and the data along with criteria for individual patient evaluation are 
available at wnw.oubscedu/platelets. Our patient met 3 out of the 4 criteria, suggestive of probable DITP.

Flow cytometry is a highly sensitive technique used for the detection of platelet-reactive antibodies induced by several drugs. Other tests include the platelet immunofluorescence test, enzyme-linked immunospecific assay and immunoprecipitation-Western blotting. Laboratory tests should be done to check for infectious causes of ITP i.e. hepatitis B and C, HIV and H. pylori. Coombs' test is done to rule out Evans' syndrome. The conditions like SLE and multiple myeloma should also be considered. Bone marrow examination can be reserved for those who do not respond to therapy, or those who have features not corresponding to ITP.

The complete withdrawal of the drug is the first step in treatment of drug induced ITP. The recovery of thrombocytopenia occurs within 1 to 2 days of discontinuation of the drug. Platelet transfusions are indicated in cases of severe thrombocytopenia (wet purpura) due to high risk of bleeding. The role of corticosteroids is controversial. ${ }^{15}$ Intravenous Ig and plasma exchange have been tried in cases of persistent thrombocytopenia and bleeding with no proven benefit. ${ }^{16,17}$

Etoricoxib is a non-steroidal anti-inflammatory drug (NSAID), belonging to the category of selective COX2 inhibitors. COX-2 is involved in the production of irritant substances in the body in response to disease and injury; and by blocking its action, etoricoxib reduces the symptoms of pain and inflammation. It is commonly used in conditions like arthritis, back pain, ankylosing spondylitis and gout. The incidence of myocardial infarction and thrombotic cerebrovascular accident have been reported to be higher with etoricoxib. ${ }^{1-3}$ Other common adverse effects include gastric irritation, hypertension and pretibial oedema and erythema. ${ }^{3,4}$ Thrombocytopenia following etoricoxib intake is a rare side effect.

\section{CONCLUSION}

Etoricoxib, a COX-2 inhibitor, is used as a pain killer in conditions like arthritis etc. Its gastrointestinal tolerance is better compared to other NSAIDs. Hypertension, pretibial oedema, myocardial infarction and thrombotic stroke are some of the noted events with etoricoxib. However, thrombocytopenia is an uncommon complication, and cases of severe DITP with wet purpura following etoricoxib intake have not been reported previously. This report also highlights the need to monitor the platelet counts of patients on long term therapy with etoricoxib for arthritis and gout.

\section{REFERENCES}

1. Haag MD, Bos MJ, Hofman A, Koudstaal PJ, Breteler MM and Stricker $\mathrm{BH}$. Cyclooxygenase selectivity of nonsteroidal antiinflammatory drugs and risk of stroke. Arch Intern Med 2008; 168(11):1219-1224.

2. Chen LC and Ashcroft DM. Risk of myocardial infarction associated with selective COX-2 inhibitors: meta-analysis of randomised controlled trials. Pharmacoepidemiol Drug Saf 2007;16(7):762-772.

3. Turajane T, Wongbunnak R, Patcharatrakul T, Ratansumawong K, Poigampetch $Y$ and Songpatanasilp T. Gastrointestinal and cardiovascular risk of non-selective NSAIDs and COX-2 inhibitors in elderly patients with knee osteoarthritis. J Med Assoc Thai 2009; 92 Suppl 6:S19-S26.

4. Kumar P. Etoricoxib-induced pretibial erythema and edema. Indian Dermatol Online J 2015; 6(Suppl 1):S47-S49.

5. Barbara AK. Disorders of platelets and vessel wall. In: Kasper, Fauci, Hauser, Longo, Jameson, Loscalzo eds. Harrison's principles of internal medicine. 19th ed. McGraw Hill education 2015, 725-729.

6. Renu S, Hara PP and Manoranjan M. de Gruchy's clinical haematology in medical practice. 6th ed. 349-359.

7. Davoren A and Aster RH. Heparin-induced thrombocytopenia and thrombosis. Am J Hematol 2006; 81:36-44.

8. Shulman NR. A Mechanism of Cell Destruction in Individuals Sensitized to Foreign Antigens and Its Implications in AutoImmunity. Combined Clinical Staff Conference at the National Institutes of Health. Ann Intern Med 1964; 60:506-521.

9. Murphy MF, Riordan T, Minchinton RM, Chapman JF, Amess JA, Shaw EJ, et al. Demonstration of an immunemediated mechanism of penicillin-induced neutropenia and thrombocytopenia. Br J Haematol 1983; 55:155-160.

10. Bougie DW, Wilker PR, Wuitschick ED, Curtis BR, Malik M, Levine $S$, et al. Acute thrombocytopenia after treatment with tirofiban or eptifibatide is associated with antibodies specific for ligand-occupied GPIIb/IIla. Blood 2002; 100:2071-2076.

11. Aster $\mathrm{RH}$. Can drugs cause autoimmune thrombocytopenic purpura? Semin Hematol 2000; 37:229-238.

12. Carey PJ. Drug-induced myelosuppression: diagnosis and management. Drug Saf 2003; 26:691-706.

13. Majhail NS and Lichtin AE. What is the best way to determine if thrombocytopenia in a patient on multiple medications is druginduced? Cleve Clin J Med 2002; 69:259-262.

14. Eisner EV and Crowell EB. Hydrochlorothiazide-dependent thrombocytopenia due to IgM antibody. JAMA 1971; 215:480-482.

15. George JN and Aster RH. Drug-induced thrombocytopenia: pathogenesis, evaluation, and management. Hematology Am Soc Hematol Educ Program 2009:153-158.

16. Ray JB, Brereton WF and Nullet FR. Intravenous immune globulin for the treatment of presumed quinidine-induced thrombocytopenia. DICP 1990; 24:693-695.

17. Pourrat $\mathrm{O}$. Treatment of drug-related diseases by plasma exchanges. Ann Med Interne (Paris) 1994; 145:357-360. 